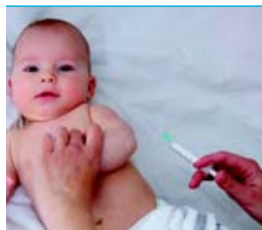

\title{
Pertussis control: where to now?
}

\section{Improving protection against pertussis requires sorting the facts from the artefacts}

ertussis is a disease of significant morbidity and, in infants, mortality. Regrettably, even though there is greater than 20 -fold reduction in pertussis burden with immunisation, ${ }^{1}$ it persists globally as a significant public health problem. For more than two decades, Australia has had the highest reported rates of pertussis in the world. ${ }^{2}$ In the 1990s, this was driven by the introduction of mandatory reporting by laboratories of positive test results for vaccine-preventable diseases to the National Notifiable Diseases Surveillance System and extensive use of serological tests for diagnosis, primarily in adults. ${ }^{3}$ Unlike many other countries, all positive test results in Australia are included in national data. Also, testing for pertussis by polymerase chain reaction (PCR) has qualified for reimbursement since 2008, after which a sevenfold increase in testing of children in general practice was documented. ${ }^{4}$ Pertussis epidemics occurred sequentially across Australia from 2008 to 2012 and, unlike previous epidemics, the highest notification rates were for children under 10 years of age. This raises the question of whether Australia's "pertussis problem" is related to vaccines with poor effectiveness or is an artefact of testing.

Observational methods are used to measure vaccine effectiveness (VE) (also known as "field efficacy"). The screening method enables estimation of VE if the vaccination status of patients with a case of the disease and population vaccine coverage are known - the more effective the vaccine, the lower the likelihood of patients with a case of the disease having been vaccinated compared with the source population. ${ }^{5}$ The screening method performs best when about $50 \%$ of the population is vaccinated. When vaccine coverage is over $90 \%$, estimates of $\mathrm{VE}$ change substantially with small changes in population coverage estimates. In this issue of the Journal, Sheridan and colleagues use the screening method to estimate VE for acellular pertussis vaccine in Queensland children during an epidemic in 2009 and $2010 .^{6}$ They found that VE for three doses in children aged from 1 to $<4$ years was over $80 \%$. However, similar to studies in the United States, ${ }^{7,8}$ VE fell significantly and progressively in children over 5 years of age, whether they had received four or five doses. It was previously reported that among Queensland children born in 1998, those who had received one or more doses of whole-cell pertussis vaccine were significantly better protected than those who had received only acellular vaccine, especially after 6 years of age. ${ }^{9}$ A national study, which included Queensland data from 2009, took a different approach - cases were individually matched by birth date to children on the Australian Childhood Immunisation Register and were limited to children younger than 4 years. ${ }^{10}$ Similar VE estimates were obtained for the first 2 years of life, but, in contrast to findings from the Queensland study, there was a significant and progressive fall in VE between ages 2 years and 4 years (the latter being the age at which children were eligible for the fourth dose).
Peter B McIntyre PhD, FRACP, FAFPHM Director ${ }^{1}$

Terence M Nolan PhD, FRACP, FAFPHM,
Head $^{2}$

1 National Centre fo Immunisation Research and Surveillance, Sydney Children's Hospitals Network Sydney, NSW.
Sydnetion,

2 School of Population and Global Health University of Melbourne, Melbourne, VIC. t.nolan@ unimelb.edu.au

doi: 10.5694/mjal4.00234 
Importantly, Sheridan et al were also able to evaluate testing patterns by age, showing that the overall number of PCR tests increased in the second year of the Queensland epidemic. ${ }^{6}$ Also, in children aged over 5 years, although PCR tests were less commonly performed, the results were more commonly positive. ${ }^{6}$ This is probably due to older children with cough being less likely to present to general practice and less likely to be tested, suggesting that notification rates of pertussis would have been even higher if more testing had been done. Disease severity is also an important consideration: assessing the disease burden from pertussis cases in older children is valuable, and VE is expected to be lower for less severe illness. ${ }^{5}$ Apart from the requirement for hospitalisation, against which VE was high for children younger than 1 year ${ }^{3}$ and $1-4$ years, ${ }^{10}$ few data on severity are available. In a recent New South Wales study using linked hospitalisation and pertussis notification data, it was found that only $2 \%$ of children over 5 years who had pertussis were hospitalised, but $8 \%$ had been taken to an emergency department. $^{11}$

What conclusions can we draw from these studies? First, the current acellular vaccines are highly effective in preventing severe pertussis, especially in the first 2 years of life, but effectiveness progressively wanes from 2 years after the last dose. Such rapid waning was not expected when the decision to forego the 18-month booster in favour of a booster for adolescents was made in 2003. This decision was based on favourable results from modelling this change using the only available data at the time ${ }^{12}$ - data which suggested that three doses provided protection up to 7 years of age, ${ }^{13}$ which contrasts with more recent findings. Australian data showing low levels of population antibody to pertussis toxin preceding the recent epidemic support the idea that the schedule change had a negative impact. ${ }^{14}$ Second, high levels of laboratory testing inflated Australian case numbers disproportionately to other countries, through identifying more ambulatory cases in children and adults. Third, pertussis vaccine coverage has increased dramatically in Australia since the epidemic in the late 1990s, with better acceptance by parents and doctors of the acellular vaccines compared with more reactogenic whole-cell vaccines. Notably, the national epidemic from 2008 to 2012 was associated with fewer deaths than the late 1990s epidemic, despite much higher numbers of cases.

Where does the future lie for pertussis vaccines in terms of improving disease control, especially death and severe morbidity? A vaccine that effectively reduces transmission and disease is an important objective for herd immunity. In this regard, there is promise from research on live attenuated vaccines, ${ }^{15}$ and the potential for acellular vaccines with improved adjuvants and less reactogenic whole-cell vaccines, but all are some years away. Immunising mothers during the last 8 weeks of pregnancy with adult-formulated acellular pertussis vaccine could prevent early infant mortality and morbidity. Reinstalling the 18-month booster in the National Immunisation Program could improve control in early childhood, if cost-effectiveness criteria can be met. For all vaccines on the National Immunisation Program, ongoing monitoring of VE is crucial and greater use of Australia's high-quality data systems can support this, as recommended in the National Immunisation Strategy. ${ }^{16}$

Competing interests: We are principal investigators in a study of birth-dose pertussis vaccination which has been partly funded by GlaxoSmithKline.

Provenance: Commissioned; externally peer reviewed.

1 Cherry JD. Epidemic pertussis in 2012 - the resurgence of a vaccinepreventable disease. NEngl J Med 2012; 367: 785-787.

2 McIntyre PB. Is Australia the world capital of pertussis? National Pertussis Workshop; 2011 Aug 25-26; Sydney, Australia. http://ncirs.edu.au/news/pastnews-events/Day\%201/Mclntyre-Is-Australia-world-capital-PertussisWS-25 26Augl1.pdf (accessed Feb 2014).

3 Quinn HE, McIntyre PB. Pertussis epidemiology in Australia over the decade 1995-2005 - trends by region and age group. Commun Dis Intell Q Rep 2007; 31: 205-215.

4 Kaczmarek MC, Valenti L, Kelly HA, et al. Sevenfold increase in likelihood of pertussis test requests in a stable set of Australian general practice encounters, 2000-2011. Med J Aust 2013; 198: 624-628.

5 Farrington CP. Estimation of vaccine effectiveness using the screening method. Int J Epidemiol 1993; 22: 742-746.

6 Sheridan SL, McCall BJ, Davis CA, et al. Acellular pertussis vaccine effectiveness for children during the 2009-2010 pertussis epidemic in Queensland. Med J Aust 2014; 200: 334-338.

7 Klein NP, Bartlett J, Rowhani-Rahbar A, et al. Waning protection after fifth dose of acellular pertussis vaccine in children. NEngl J Med 2012; 367: 1012-1019.

8 Misegades LK, Winter K, Harriman K, et al. Association of childhood pertussis with receipt of 5 doses of pertussis vaccine by time since last vaccine dose, California, 2010. JAMA 2012; 308: 2126-2132.

9 Sheridan SL, Ware RS, Grimwood K, Lambert SB. Number and order of whole cell pertussis vaccines in infancy and disease protection. JAMA 2012; 308: 454-456.

10 Quinn HE, Snelling TL, Macartney KK, McIntyre PB. Duration of protection after first dose of acellular pertussis vaccine in infants. Pediatrics 2014; 133: e513-e519.

11 McCallum LK, Liu B, McIntyre P, Jorm LR. Estimating the burden of pertussis in young children on hospitals and emergency departments: a study using linked routinely collected data. Epidemiol Infect 2014; 142: 695-705.

12 Hethcote HW, Horby P, McIntyre P. Using computer simulations to compare pertussis vaccination strategies in Australia. Vaccine 2004; 22: 2181-2191.

13 Salmaso S, Mastrantonio P, Tozzi AE, et al. Sustained efficacy during the first 6 years of life of 3-component acellular pertussis vaccines administered in infancy: the Italian experience. Pediatrics 2001; 108: E81.

14 Campbell P, McIntyre P, Quinn $\mathrm{H}$, et al. Increased population prevalence of low pertussis toxin antibody levels in young children preceding a record pertussis epidemic in Australia. PLOS ONE 2012; 7: e35874.

15 Thorstensson R, Trollfors B, Al-Tawil N, et al. A phase I clinical study of a live attenuated Bordetella pertussis vaccine - BPZEl; a single centre, double-blind, placebo-controlled, dose-escalating study of BPZEl given intranasally to healthy adult male volunteers. PLOS ONE 2014; 9: e83449.

16 Australian Government Department of Health. National Immunisation Strategy 2013-2018. Canberra: DOH, 2013. http://www.immunise.health. gov.au/internet/immunise/publishing.nsf/Content/A894B6DF512D815DCA 257BA300053819/\$File/nat-immune-strategy-2013-18-final.pdf (accessed Mar 2014). 